\title{
Mid-Infrared Spectral Assessment of Early-type Galaxies with Star Formation
}

\section{Lerothodi L. Leeuw ${ }^{1,2}$, Jesse D. Bregman ${ }^{3}$, Jacqueline Davidson $^{4}$, Pasquale Temi ${ }^{3}$ and Stephen S. $\mathrm{Im}^{3}$}

\author{
${ }^{1}$ Physics Department, U. of Johannesburg, P.O. Box 524, Auckland Park, 2006, South Africa \\ email: lerothodi@alum.mit.edu \\ ${ }^{2}$ SETI Institute, 515 N. Whisman Avenue, Mountain View, CA 94043, USA \\ ${ }^{3}$ Space Science and Astrophysics Branch, NASA Ames Research Center, MS 245-6, \\ Moffett Field, CA 94035, USA \\ ${ }^{4}$ U. of Western Australia, School of Physics, 35 Stirling Hwy, Crawley, WA 6009, Australia
}

\begin{abstract}
Mid-infrared (mid-IR) spectra from $\sim 5$ to $14 \mu \mathrm{m}$ of five, nearby $(<70 \mathrm{Mpc}$ ) elliptical galaxies are presented that were observed with the Infrared Spectrograph on the Spitzer Space Telescope. The sample galaxies have a main stellar component that is typical for normal, passively evolving ellipticals; however, they are rich in cold gas and dust and have morphologicalmerger signatures from which a time order of the galaxies since the merger or accretion events can be estimated. The presented results are significant because (1) emission due to Polycyclic Aromatic Hydrocarbons (PAHs) and associated species is detected for the first time in these galaxies and (2) the detected mid-IR spectra are independently exploited as a probe of current or recent star-formation that, in this case, is assumed to be triggered by the merger. As shown in exemplary spectra of the early-age merger NGC 3656, the strength of the PAH emission is more centrally peaked in the earlier-age mergers, suggesting that the PAH data are indeed probing star-formation that is correlated with the time since the mergers and systematically depletes the centrally located gas, becoming weaker and more flatly distributed as the merger evolves.
\end{abstract}

Keywords. galaxies: elliptical and lenticular, cD - galaxies: ISM - galaxies: photometry infrared: galaxies

\section{Introduction}

The mid-infrared (mid-IR) $6.2 / 11.3 \mu \mathrm{m}$ and $7.7 / 11.3 \mu \mathrm{m}$ intensity ratios of Polycyclic Aromatic Hydrocarbons (PAHs) have been shown to be correlated with star-formation intensity in local regions within the nearby starburst galaxy M 82 (e.g., Galliano et al. 2008) and the merger remnant galaxy Centaurus A (e.g., Quillen et al. 2008). The present paper briefly presents an illustration of how these ratios can be used to assess starformation in exemplary data of NGC 3656, the earliest-age merger galaxy in our sample of nearby elliptical galaxies that were known to have CO in centrally located gas disks (Young et al. 2009; Leeuw et al. 2008 and references therein) and morphological merger tracers. The data demonstrate the usefulness of the ratios in elucidating evolution of or, at least, delineating ionization due to star-formation in these ellipticals. Other observational evidence of star-formation in these galaxies was presented by Leeuw et al. (2008), using $350 \mu \mathrm{m}$ continuum data, and confirmed for some of the galaxies by Young et al. (2009), using 24 to $160 \mu \mathrm{m}$ continuum data.

The galaxies in our sample are all ellipticals in that their luminosity profiles follow the de Vaucoleur, $\mathrm{r}^{1 / 4}$ law (e.g., Rothberg \& Joseph 2004; Young 2005); however, they represent a spread of merger tracers or ages, from galaxies that have been morphologicaly classified as on-going or early-age major mergers to very-late accretion or quiescent 
systems (see Table 1). The description of the sample as ellipticals with merger or accretion signatures and star-formation (as we have now shown), or mergers with elliptical components, is consistent with the very last stages of an evolution of mergers to form elliptical galaxies or ellipticals that are in the final stages of relaxation after an accretion or merger event with a gas-rich companion.

Table 1. Published optical and molecular-gas properties of the ellipticals (adopted from Leeuw et al. 2008 and references therein)

\begin{tabular}{lccccccc}
\hline Source & Type & Environment, Notables, \& & $M\left(\mathrm{H}_{2}\right)$ & $M(\mathrm{HI})$ & $D$ & $L_{B}$ & $(B-$ \\
& & Merger or Accretion Stage & {$\left[10^{8}\right.$} & {$\left[10^{8}\right.$} & {$[\mathrm{M}$} & {$\left[10^{9}\right.$} & $V)_{e}$ \\
& & $\left.\mathrm{M}_{\odot}\right]$ & $\left.\mathrm{M}_{\odot}\right]$ & $\mathrm{pc}]$ & $\left.\mathrm{L}_{\odot}\right]$ & mag \\
\hline NGC 3656 & Ep & Early-Age Major Merger/Accr.? & 47 & 11 & 45 & 16 & \\
NGC 5666 & $\mathrm{cE2/SO}$ & Intermediate Merger/Accr.? & 5.7 & 10 & 35 & 6 & 0.86 \\
UGC 1503 & $\mathrm{E} 1$ & Intermediate-to-Late Merger/Accr.? & 18 & 16 & 69 & 16 & \\
NGC 0759 & E0/1 & Cluster A262, Late Merger/Accr.? & 24 & $<24$ & 66 & 34 & 1.05 \\
NGC 0807 & E3 & Field, Quiescent / Very-Late Accr.? & 14 & 61 & 64 & 31 & 0.97 \\
\hline
\end{tabular}

\section{Spitzer Infrared Spectrograph Observations and Results}

Our observing objective was to obtain spectra of the mid-IR PAH features and continuum for the sample early-type galaxies (see Table 1) and investigate their relation to the star-formation activity in their central dusty-gas disks of $\sim 5 \mathrm{kpc}$ radii (Young et al. 2009; Leeuw et al. 2008 and references therein). If star-formation is taking place, strong emission from PAH and emission lines, well above the mid-IR continuum, is expected (e.g., Bregman et al. 2006). To achieve our observing objective, we used the 5.2 -to- $14.0 \mu \mathrm{m}$ Short-Low 1st and 2nd order configurations of Spitzer Space Telescope (Spitzer) Infrared Spectrograph (IRS) in staring mode. The pointing was centered on the galaxy peak, allowing the IRS slit dimensions of $57^{\prime \prime} \times 3 .^{\prime \prime} 7$ to cover both the CO and cold-dust emission-rich regions in the center and emission-poor regions in the outer parts of each galaxy. The data were obtained in our Spitzer General Observer Program 30444.

Because the observed mid-IR emission is extended in most of the galaxies, mid-IR spectra have been extracted in fixed-column extractions that are 4-pixel- or $7 .^{\prime \prime} 2$-wide along the slit, for locations at the center and 4 pixels to 12 pixels left and right of the center when the extractions can be obtained at high significance. The spectra from all the galaxies show strong PAH emission in the four main-PAH bands at 6.2, 7.7, 8.6, and $11.3 \mu \mathrm{m}$ and minor-PAH bands at $12.7 \mu \mathrm{m}$ as well as weaker 5.7, 12.0, and $14.2 \mu \mathrm{m}$ PAH bands. In some regions of the galaxies there are also rotational molecular hydrogen lines and forbidden emission lines. Integrated fluxes in the four main-PAH bands were recovered after substracting a cubic-spline continuum of the galaxies, following the methods of Peeters, Spoon \& Tielens (2004). The strengths of the four main PAH bands and also their ratios vary among and also for different locations within the galaxies. Some of the spectral details, variations, and their implications are discussed in below, high-lighting the results from the early-merger remnant NGC 3656 and then concluding with a summary for the whole sample.

\subsection{The Early-Age Major-Merger/Accretion Elliptical NGC 3656}

Of our sample galaxies, NGC 3656 shows the clearest morphological signatures of a past interaction. One such sign was presented by Balcells et al. (2001) in terms of resolved 
imaging of H I in shells and tidal tails of this galaxy. The H I is more extended than the galaxy's main stellar elliptical and dusty-gas disk components. Sensitive CCD optical images of the dust features in NGC 3656 were studied by Balcells (1997) and Rothberg \& Joseph (2006), who suggested that the dust is a remnant of a dust-rich merger that involved this galaxy's primary optical elliptical component.

Figure 1 shows spectra from five regions that are 4-pixel-wide (or $1.6 \mathrm{kpc}$-wide at $\mathrm{D}=45 \mathrm{Mpc}$ ) in NGC 3656. As labeled in this figure, the central region and those around it are plotted for both Nod-One and -Two IRS observation positions, while those farther out are only available and plotted for one nod positions. The spectra from the central region of NGC 3656 is unique to other regions observed for the entire sample in that it has very strong 6.2 and $7.7 \mu \mathrm{m}$ PAH bands and [NeII] $(12.8 \mu \mathrm{m})$ emission lines, with the strength of the $7.7 \mu \mathrm{m}$ PAH band and [Ne II] emission line actually higher than that of the $11.3 \mu \mathrm{m}$ PAH band. Such strong 7.7 $\mu \mathrm{m}$ PAH bands and [NeII] emission lines are typical of star-formation galaxies and independently confirm that star-formation is particularly strong in the central region of this galaxy, as was reported by Leeuw et al. 2008 and Young et al. 2009 respectively based on submm $350 \mu \mathrm{m}$ and Spitzer far-infrared 24, 70 , and $160 \mu \mathrm{m}$ observations. As noted above, these spectra also show several rotational molecular hydrogen and forbidden emission lines and some minor PAH bands.

The top and second-bottom panels in Figure 1 show that spectra from the regions $7 .{ }^{\prime \prime} 2$ on either side of the center also have fairly strong PAH bands. However, the strengths of the 6.2 and $7.7 \mu \mathrm{m} \mathrm{PAH} \mathrm{bands} \mathrm{are} \mathrm{much} \mathrm{weaker} \mathrm{relative} \mathrm{to} 11.3 \mu \mathrm{m} \mathrm{PAH} \mathrm{band} \mathrm{and}$ their respective strengths in the central region. The $6.2 / 11.3 \mu \mathrm{m}$ and $7.7 / 11.3 \mu \mathrm{m} \mathrm{PAH}$ band ratios in these regions are also smaller than in the center, and continue falling with distance outward, suggesting that star-formation, or at least the ionizing radiation related to it, in NGC 3656, also falls with distance away from the galactic center (Leeuw et al. in preparation). The spectra from the $7 .^{\prime \prime} 2$-wide region centered 4 pixels (or $1.6 \mathrm{kpc}$ ) northeast or left of the galactic center of NGC 3656 along the IRS slit position has a spectral shape that rises longward of $10 \mu \mathrm{m}$ and exhibits strong [Ne II] and [Ne V] forbidden emission lines that are indicative of H II regions. As is typical of H II regions, the rising spectrum is also an indication that the dust in this region is not only composed of large, cold grains that are thermally heated (Leeuw et al. 2008) but also small grains that are stochastically heated by intense radiation in this region. The presence of small grains here can also be inferred from the broader $8.6 \mu \mathrm{m}$ band seen this spectrum. The discovery of an H II region in this early-type galaxy shows the value of the presented IRS spectra and the regional spectral extractions we have made.

\section{Summary of Results and Conclusions}

The presented Spitzer spectral $\sim 5$ to $14 \mu \mathrm{m}$ results are significant because (1) emission due to PAHs and associated species is detected for the first time in these gas-rich earlytype galaxies and (2) the detected mid-IR spectra have been independently exploited as a probe of current or recent star-formation that, in this case, is assumed to be triggered by mergers whose signatures are present in the studied galaxies. The strength of the PAH emission is more centrally peaked in the earlier-age mergers (e.g. NGC 3656), suggesting that the PAH data are probing star-formation that is correlated with the time since the mergers and systematically depletes the centrally located gas, becoming weaker and more flatly distributed as the merger evolves. The correlation of morphological merger indicators with star-formation intensity, as indicated by PAH intensities and their ratios, can be investigated further with a larger sample of ellipticals, by exploiting data from different guest observer programs available in the Spitzer Data Archive. An extended 
mid-IR assessment of star-formation in local regions of nearby elliptical galaxies should also be possible with higher resolution imaging that is available with instruments on the Herschel Space Observatory and SOFIA, as well as some 4- and 10-m class ground based telescopes.

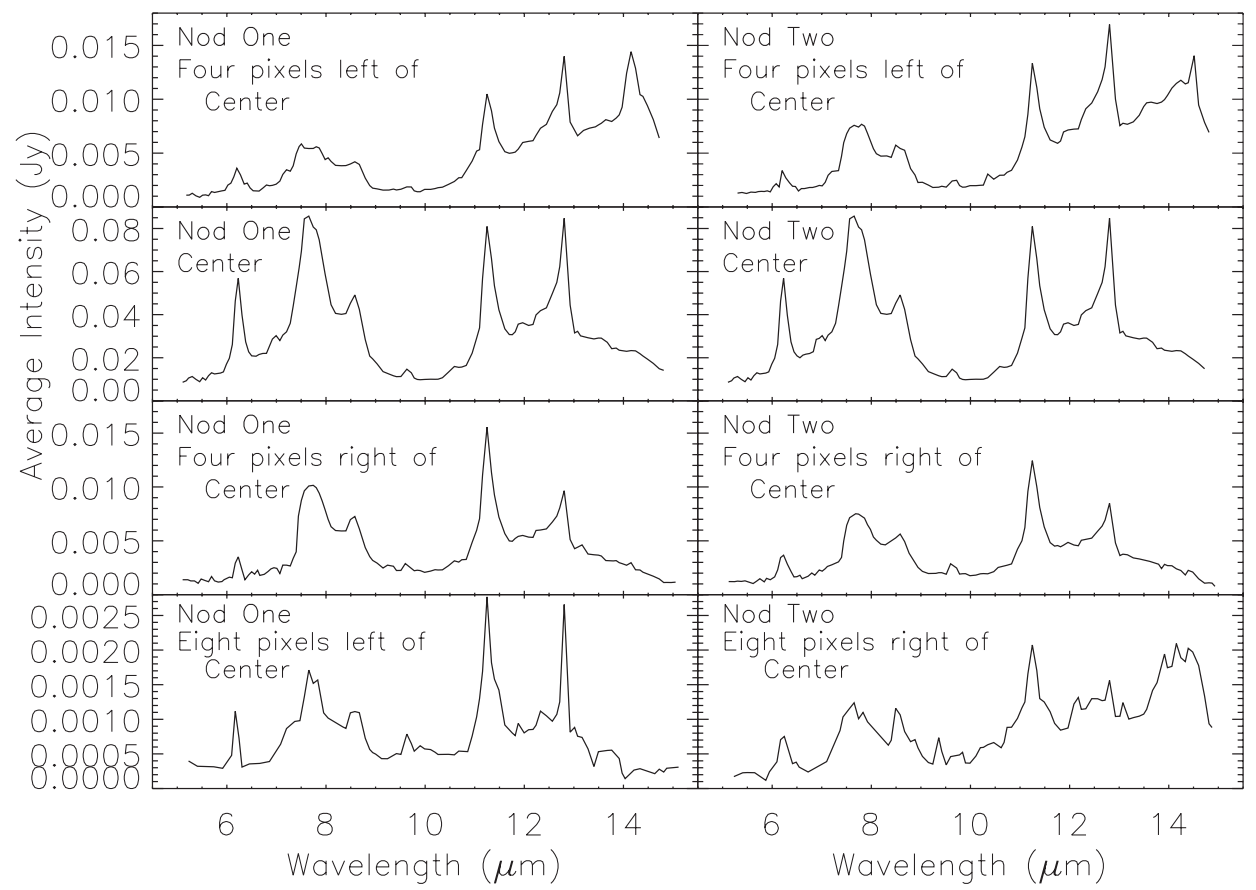

Figure 1. Spectra of 4-pixel-wide regions across NGC 3656. Left column are nod one, and right column are nod two spectra. The 1st row is from spectra centered at 4 pixels left of the mid-IR peak (or galactic center); the 2nd row at the center; the 3rd row 4 pixels right of the center; the 4 th row, 1st column 8 pixels left of the center; and the 4th row, 2nd column 8 pixels right of the center. Positions "right of center" are southwest and "left of center" are northeast of the galactic center.

\section{References}

Balcells, M. 1997, ApJL, 486, L87

Balcells, M., van Gorkom, J. H., Sancisi, R., \& del Burgo, C. 2001, AJ, 122, 1758

Bregman, J. N., Temi, P., \& Bregman, J. D. 2006, ApJ, 647, 265

Galliano, F., Madden, S. C., Tielens, A. G. G. M., Peeters, E., \& Jones, A. P. 2008, ApJ, 679, 310

Leeuw, L. L., Davidson, J., Dowell, C. D., \& Matthews, H. E. 2008, ApJ, 677, 249

Quillen, A. C., Bland-Hawthorn, J., Green, J. D., Smith, J. D., Prasad, D. A., Alonso-Herrero, A., Cleary, K., Brookes, M. H., \& Lawrence, C. R. 2008, MNRAS, 384, 1469

Rothberg, B. \& Joseph, R. D. 2004, AJ, 128, 2098

—. 2006, AJ, 132, 976727

Young, L. M. 2005, ApJ, 634, 258

Young, L. M., Bendo, G. J., \& Lucero, D. M. 2009, AJ, 137, 3053 